Publ. Mat. (2007), 119-130

Proceedings of the Primeras Jornadas de Teoría de Números.

\title{
REGULATORS AND TOTAL POSITIVITY
}

\section{EDUARDO FRIEDMAN}

Abstract

The classical regulator $R$ of a number field $K$ is given $[\mathbf{F} \mathbf{1}]$ by a rapidly convergent series of the form

$$
\frac{R}{w}=\sum_{m=1}^{\infty} a_{m} g\left(m^{2} /|D|\right),
$$

where $w$ is the number of roots of unity in $K, D$ is the discriminant of $K, a_{m}$ counts certain integral ideals in $K$ of absolute norm $m$, and $g:(0, \infty) \mapsto \mathbb{R}$ is defined as

$g(x):=\frac{1}{2^{r_{1}} 4 \pi i} \int_{2-i \infty}^{2+i \infty}\left(4^{r_{2}} \pi^{[K: \mathbb{Q}]} x\right)^{-s / 2}(2 s-1) \Gamma\left(\frac{s}{2}\right)^{r_{1}} \Gamma(s)^{r_{2}} d s$, $r_{1}$ and $r_{2}$ being, respectively, the number of real and complex places of $K$. If the unit group of $K$ is infinite, it is known that $g(x)$ tends to $-\infty$ as $x \rightarrow 0^{+}$, and that $g(x)$ is positive and vanishes exponentially fast for large $x$. Using classical results from the theory of total positivity we prove that $g$ has the simplest possible behavior compatible with these asymptotic data. Namely, $g(x)$ has a unique zero in $(0, \infty)$, and the same holds for each derivative of $g$. This leads to a new lower bound for the regulator

$$
R>w g(1 /|D|),
$$

which is useful for certain ranges of $D$.

\section{Introduction}

In the 1930's Remak set about proving lower bounds for the regulator of a number field. Using the geometry of numbers he was able to prove $[\mathbf{R e} 1]$ for totally real fields $K$

$$
R \geq c_{0} c_{1}^{n},
$$

where $R$ denotes the classical unit regulator of $K, c_{0}>0$ and $c_{1}>1$ are explicit absolute constants, and $n:=[K: \mathbb{Q}]$. If $K$ is now a general

2000 Mathematics Subject Classification. Primary: 11R27; Secondary: 11R42.

Key words. Regulator, total positivity.

This work was partially supported by Chilean Fondecyt grant number 1040585. 
number field, but not a totally complex quadratic extension of a totally real field, he proved $[\mathbf{R e} 2]$

$$
R \geq C_{1} \log \left(|D| / n^{n}\right),
$$

where $D$ is the discriminant of $K$ and $C_{1}$ is some explicit positive constant depending on $n$ (as are all $C_{i}$ below). For number fields without proper subfields, Remak $[\mathbf{R e 2}]$ proved the better bound

$$
R \geq C_{2} \cdot\left(\log \left(|D| / n^{n}\right)\right)^{\rho_{K}},
$$

where $\rho_{K}$ is the unit-rank of $K$. Silverman [Si1] generalized this in 1984 to arbitrary number fields, proving

$$
R \geq C_{3} \cdot\left(\log \left(|D| / C_{4}\right)\right)^{\rho_{K}-\rho_{k}},
$$

where $k$ is a proper subfield of $K$ having maximal unit-rank.

Remak deduced from (1.1) and (1.2) that there was a smallest regulator among all totally real number fields and showed that this minimum was at least $10^{-3}$. In 1978 Pohst $[\mathbf{P o}]$ considerably improved on the values of $c_{0}$ and $c_{1}$ in (1.1), coming very close to proving that the minimal regulator for totally real fields corresponds to $K=\mathbb{Q}(\sqrt{5})$.

In 1981 Zimmert [ $\mathbf{Z i}$ ] introduced analytic techniques that yielded (1.1) for number fields of any signature. Using his improved bounds he was able to complete Pohst's work and show that the minimal discriminant among totally real fields is indeed that of $\mathbb{Q}(\sqrt{5})$.

In 1989, using a related analytic method, I proved a formula for the regulator. Namely $[\mathbf{F 1}$, Theorem A],

$$
\frac{R}{w}=\sum_{\mathfrak{a}} g\left(\mathrm{Na}^{2} /|D|\right)+\sum_{\mathfrak{b}} g\left(\mathrm{Nb}^{2} /|D|\right),
$$

where $w$ is the number of roots of unity in $K, \mathfrak{a}$ runs over the principal integral ideals of $K, \mathfrak{b}$ runs over the integral ideals of $K$ in the ideal class of the different of $K, \mathrm{~N}$ denotes the norm, and $g:(0, \infty) \mapsto \mathbb{R}$ is given by

$$
\text { (1.4) } \begin{aligned}
g(x)= & g_{r_{1}, r_{2}}(x) \\
& :=\frac{1}{2^{r_{1}} 4 \pi i} \int_{2-i \infty}^{2+i \infty}\left(\pi^{n} 4^{r_{2}} x\right)^{-s / 2}(2 s-1) \Gamma\left(\frac{s}{2}\right)^{r_{1}} \Gamma(s)^{r_{2}} d s,
\end{aligned}
$$

with $r_{1}$ and $r_{2}$ denoting the number of real and complex places of $K$, respectively.

To compute $R / w$ from (1.3) requires calculating $g$ at least for all terms $\mathfrak{a}$ and $\mathfrak{b}$ having norm smaller than $\sqrt{|D|}$. Although all proven methods of computing $R$ are roughly of this complexity, in practice it is 
faster to use Buchmann's algorithm [Co1, §6.5]. In fact, the main application of the regulator formula (1.3) is to obtain lower bounds for $R / w$. We shall prove

Theorem 1. Let $R$ and $w$ denote, respectively, the regulator and the number of roots of unity in a number field $K$ of discriminant $D$. Then $R / w>g(1 /|D|)$, where $g$ is given by (1.4) above.

We hasten to add that $g$ takes both positive and negative values. In fact [F1, p. 609, Remark iv], if $r_{1}+r_{2} \geq 2$,

$$
\lim _{x \rightarrow 0^{+}} g(x)=-\infty .
$$

Thus, for each signature $\left(r_{1}, r_{2}\right)$, Theorem 1 is useful only for a finite number of fields.

The new analytic result underlying Theorem 1 is

Theorem 2. The $C^{\infty}$-function $g:(0, \infty) \mapsto \mathbb{R}$ defined in (1.4) has a unique zero, and this zero is simple. Moreover, for every integer $k \geq 1$, the same statement holds for the $k$-th derivative of $g$.

It follows that $g$ is unimodal, as are all of its derivatives.

We shall deduce Theorem 2 from Schoenberg's 1950 study of convolution transforms associated to Pólya frequency functions $[\mathbf{S c h}]$. These interesting results are part of the wider theory of total positivity, which is admirably developed in Karlin's treatise [Ka]. In Section 2 we give an account of what we need from this theory. In Section 3 we give the proof of Theorem 2 .

Theorem 1 then follows directly. Indeed, we may (trivially) assume $g(1 /|D|)>0$. From Theorem 2 and the behavior near $x=0$ given in (1.5), we conclude that $g(x)>0$ for $x \geq 1 /|D|$. As all ideals appearing in (1.3) are integral, on dropping all terms in (1.3) other than the one corresponding to the unit ideal, we obtain Theorem 1.

In [F1] I used the behavior of $g(1 /|D|)$ for small discriminants to find the smallest regulator among all number fields. That work can now be substantially simplified using Theorem 2 , as was already observed then $[\mathbf{F 1}$, p. 605].

We give next some numerical examples showing the power and limitations of Theorem 1 . Let $K$ be the totally complex field of degree 36 with discriminant $D_{0}=3^{18} \cdot 4057^{9}$ given in [Co2, $\left.\S 12.2 .2\right]$. This field has a root discriminant only $1.7 \%$ larger than Odlyzko's lower bound, conditional on the Generalized Riemann Hypothesis [Od]. Zimmert's 
lower bound for the regulator gives $([\mathbf{Z i}$, Satz 3], with $\gamma=0.336)$

$$
\frac{R}{w}>121 \text {. }
$$

Theorem 1 gives approximatively

$$
\frac{R}{w}>g\left(1 / D_{0}\right) \approx 27839 .
$$

PARI $[$ Co2, $\S 11.2]$ calculates

$$
\frac{R}{w} \approx 172495 \text {. }
$$

For fields of the same signature and larger discriminant, the bounds get considerably better, up to a point. For example, using the same $D_{0}=3^{18} \cdot 4057^{9} \approx 1.15 \cdot 10^{41}$ :

$$
\begin{aligned}
& g\left(1 /\left(4 D_{0}\right)\right) \approx 47499, \\
& g\left(1 /\left(10^{8} D_{0}\right)\right) \approx 1.1 \cdot 10^{7}, \\
& g\left(1 /\left(10^{15} D_{0}\right)\right) \approx 2.2 \cdot 10^{8}, \\
& g\left(1 /\left(10^{16} D_{0}\right)\right) \approx 1.93 \cdot 10^{8}, \\
& g\left(1 /\left(10^{17} D_{0}\right)\right) \approx-3.6 \cdot 10^{7} .
\end{aligned}
$$

Thus, in signature $(0,18)$, the lower bound coming from Theorem 1 peaks at discriminants around $10^{15} D_{0}$ and becomes useless by discriminants starting about $10^{17} D_{0}$. Zimmert's bound $R / w>121$, while far worse than ours for discriminant smaller than $10^{16} D_{0}$, has the advantage of remaining valid for all totally complex fields of degree 36 .

In the above example, $K$ has a discriminant small enough to allow PARI to calculate the regulator. For somewhat larger degrees this calculation is still far out of range, so lower bounds can still be useful. For example, for the totally complex field of degree 80 and discriminant $D_{1}=257^{20} \cdot 641^{19} \approx 3.3 \cdot 10^{101}$ discovered by Martinet [Ma, Table 1] [Co2, §12.2.2], Theorem 1 gives

$$
\frac{R}{w}>4.37 \cdot 10^{15}
$$

while Zimmert's bound ([Zi, Satz 3] with $\gamma=0.214)$ is

$$
\frac{R}{w}>1.29 \cdot 10^{10} \text {. }
$$

Once again, our bound is much better for discriminants in a certain range, after which the bound quickly becomes useless. Improving on Zimmert's bounds in all discriminant ranges is still an open problem. 


\section{Total positivity}

In this section we summarize the results from the theory of total positivity required to prove Theorem 2. The first chapter of Karlin's treatise $[\mathbf{K a}]$ is an excellent introduction to the subject. We do not give theorems in their sharpest or most general form, but rather in the simplest sufficient for our purposes.

A non-negative function $K: \mathbb{R} \times \mathbb{R} \mapsto[0, \infty)$ is called totally positive of order 2 (abbreviated $\left.\mathrm{TP}_{2}\right)$ iff

$$
\operatorname{det}\left(\begin{array}{ll}
K\left(x_{1}, y_{1}\right) & K\left(x_{1}, y_{2}\right) \\
K\left(x_{2}, y_{1}\right) & K\left(x_{2}, y_{2}\right)
\end{array}\right) \geq 0
$$

whenever $x_{1}<x_{2}$ and $y_{1}<y_{2}$. An example of a $\mathrm{TP}_{2}$ function is $K(x, y)=$ $\mathrm{e}^{x y}$, as is easily verified directly from the definition.

There are a number of operations that preserve the $\mathrm{TP}_{2}$ property. If $K(x, y)$ is $\mathrm{TP}_{2}$, then so is $\hat{K}(x, y)=\varphi_{1}(x) \varphi_{2}(y) K\left(\vartheta_{1}(x), \vartheta_{2}(y)\right)$, where the $\varphi_{i}: \mathbb{R} \mapsto[0, \infty)$ are arbitrary non-negative functions and the $\vartheta_{i}: \mathbb{R} \mapsto \mathbb{R}$ are arbitrary increasing functions $(i=1,2)$. Applying this to $K(x, y)=\mathrm{e}^{x y}$, we find that

$$
k(x, y):=\exp \left(\left(-\mathrm{e}^{-x}\right)\left(\mathrm{e}^{y}\right)\right)=\exp \left(-\mathrm{e}^{y-x}\right)
$$

is $\mathrm{TP}_{2}$. Also, if $\varepsilon \in \mathbb{R}$ and if $K$ is $\mathrm{TP}_{2}$, then so is $K_{\varepsilon}(x, y):=\mathrm{e}^{\varepsilon(y-x)} K(x, y)$.

A less obvious operation preserving the $\mathrm{TP}_{2}$ property is "composition". If $K_{1}$ and $K_{2}$ are $\mathrm{TP}_{2}$ and measurable, then their composition

$$
K_{3}(x, y):=\int_{-\infty}^{\infty} K_{1}(x, t) K_{2}(t, y) d t
$$

is also $\mathrm{TP}_{2}[\mathbf{K a}, \S 1.2]$, assuming $K_{3}(x, y)$ is finite for all $x, y \in \mathbb{R}$.

Our interest in $\mathrm{TP}_{2}$ functions comes from the "variation-diminishing" property of the integral transform $q \mapsto q_{K}$, where

$$
q_{K}(x):=\int_{-\infty}^{\infty} q(y) K(x, y) d y .
$$

Briefly put, if $q$ changes sign at most once, then so does $q_{K}$.

By definition, if $I \subset \mathbb{R}$ and $q: I \rightarrow \mathbb{R}$, we shall say that $q$ changes sign at most once on $I$ iff for all triples $y_{1}<y_{2}<y_{3}$ with $y_{i} \in I$ and $q\left(y_{i}\right) \neq 0(i=1,2,3)$, we have $\operatorname{sign}\left(q\left(y_{2}\right)\right)=\operatorname{sign}\left(q\left(y_{1}\right)\right)$ or $\operatorname{sign}\left(q\left(y_{2}\right)\right)=$ $\operatorname{sign}\left(q\left(y_{3}\right)\right)$. If $I=\mathbb{R}$ we shall simply say that $q$ changes sign at most 
once. The following variation-diminishing property is due to Schoenberg and Karlin (see [Ka, §1.3]):

Suppose the $\mathrm{TP}_{2}$ function $K(x, y)$ is continuous, that $\int_{-\infty}^{\infty} K(x, y) d y$ is finite for each $x \in \mathbb{R}$, and that $q: \mathbb{R} \rightarrow \mathbb{R}$ is continuous, bounded and changes sign at most once. Then $q_{K}$ defined in (2.3) changes sign at most once.

In fact, this variation-diminishing property essentially characterizes $\mathrm{TP}_{2}$ functions [Ka, §5.3, Theorem 3.1].

We will be mainly interested in the convolution case, i.e. when the $\mathrm{TP}_{2}$ function $K$ has the form $K(x, y)=f(x-y)$ for some function $f: \mathbb{R} \mapsto[0, \infty)$. Such a function $f$ is then called a Pólya frequency function of order 2, abbreviated $\mathrm{PF}_{2}$. In this case the composition rule (2.2) states that the convolution

$$
(f * g)(x-y):=\int_{-\infty}^{\infty} f(x-y-r) g(r) d r=\int_{-\infty}^{\infty} f(x-t) g(t-y) d t
$$

of two $\mathrm{PF}_{2}$ functions is again a $\mathrm{PF}_{2}$ function (assuming $(f * g)(t)$ is finite for all real $t) . \mathrm{PF}_{2}$ functions are readily characterized $[\mathbf{K a}, \S 4.1$, Theorem 1.9]: A Lebesgue-measurable positive function $f: \mathbb{R} \mapsto(0, \infty)$ is $\mathrm{PF}_{2}$ if and only if $x \mapsto \log (f(x))$ is concave.

In the next (known) lemma we extend the variation-diminishing property to a case where $\int_{\mathbb{R}} K(x, y) d y$ is not necessarily finite.

Lemma 1. Suppose $f: \mathbb{R} \mapsto(0, \infty)$ and $q: \mathbb{R} \mapsto \mathbb{R}$ are continuous, that $f$ is $\mathrm{PF}_{2}$, and that $q$ has at most one sign change. Suppose furthermore that for some positive $C, N$ and $\gamma$ we have the bounds for $f$ and $q$ :

$$
\begin{aligned}
& f(t),|q(t)| \leq C \cdot\left(1+t^{N}\right) \quad \text { for } t \geq 0, \\
& f(t),|q(t)| \leq C \mathrm{e}^{\gamma t} \quad \text { for } t \leq 0 \text {. }
\end{aligned}
$$

Then $(f * q)(x):=\int_{-\infty}^{\infty} q(y) f(x-y) d y$ converges for all $x \in \mathbb{R}$ and $f * q$ has at most one sign change.

Proof: The convergence of the integral giving $f * q$ is immediate. For any function $g: \mathbb{R} \mapsto \mathbb{R}$, set $g_{\varepsilon}(t):=\mathrm{e}^{-\varepsilon t} g(t)$, where $\varepsilon:=\gamma / 2$. Clearly, $g$ has at most one sign change iff the same holds for $g_{\varepsilon}$. The advantage of switching from $f$ to $f_{\varepsilon}$ is that $\int_{-\infty}^{\infty} f_{\varepsilon}(x-y) d y<\infty$ for all $x \in \mathbb{R}$, and $f_{\varepsilon}(x-y)$ is still $\mathrm{TP}_{2}$ (see the remark following (2.1)). Furthermore, $q_{\varepsilon}$ is bounded and has at most one sign change. It follows from the variation-diminishing property that $f_{\varepsilon} * q_{\varepsilon}$ has at most one sign change. 
But $f_{\varepsilon} * q_{\varepsilon}=(f * q)_{\varepsilon}$. Indeed,

(2.4) $\left(f_{\varepsilon} * q_{\varepsilon}\right)(x)=\int_{-\infty}^{\infty} \mathrm{e}^{-\varepsilon(x-y)} f(x-y) \mathrm{e}^{-\varepsilon y} q(y) d y=\mathrm{e}^{-\varepsilon x}(f * q)(x)$.

Hence $f * q$ has at most one sign change.

\section{Proof of Theorem 2}

We prove here that $g=g_{r_{1}, r_{2}}$ defined in (1.4) has exactly one zero $z_{0}$ in $(0, \infty)$, that this zero is simple, and that the same holds for each derivative $g^{(k)}$. We will in fact show that the zero $z_{k}$ of $g^{(k)}$ satisfies $z_{k}>z_{k-1}(k \geq 1)$.

Using the duplication formula for the $\Gamma$-function and the change of variables $z=s / 2$, we can rewrite (1.4) as

(3.1) $g(x)=\frac{1}{2^{r_{1}}(2 \sqrt{\pi})^{r_{2}} 2 \pi i} \int_{1-i \infty}^{1+i \infty}\left(\pi^{n} x\right)^{-z}(4 z-1) \Gamma(z)^{r_{1}+r_{2}} \Gamma\left(z+\frac{1}{2}\right)^{r_{2}} d z$.

The simplest case of Theorem 2 is verified directly:

$$
\begin{aligned}
Q(x):= & g_{1,0}(x) \\
& =\frac{1}{4 \pi i} \int_{1-i \infty}^{1+i \infty}(\pi x)^{-z}(4 z-1) \Gamma(z) d z=\left(2 \pi x-\frac{1}{2}\right) \mathrm{e}^{-\pi x},
\end{aligned}
$$

where we have used the inverse Mellin transform formula

$$
\frac{1}{2 \pi i} \int_{c-i \infty}^{c+i \infty} x^{-z} \Gamma(z) d z=\mathrm{e}^{-x} \quad(c>0, x>0) .
$$

Clearly, $Q(x)$ has a unique simple zero $z_{0}$. Induction on $k$ and (3.2) yield

$$
Q^{(k)}(x):=\frac{d^{k} Q}{d x^{k}}=(-\pi)^{k}\left(2 \pi x-\left(2 k+\frac{1}{2}\right)\right) \mathrm{e}^{-\pi x} .
$$

$Q^{(k)}$ has a unique zero $z_{k}$, which moreover is simple and satisfies $z_{k}>$ $z_{k-1}$.

In proving Theorem 2 we may now assume that $n=r_{1}+2 r_{2}>1$. The theorem will follow once we show that $g^{(k)}$ is obtained from $Q^{(k)}$ by applying a variation-diminishing transform. 
Recall that if $j: \mathbb{R} \mapsto \mathbb{C}$, its (two-sided) Laplace transform is $L_{j}(z):=$ $\int_{-\infty}^{\infty} \mathrm{e}^{-z t} j(z) d z$. We shall need a simple fact about Laplace transforms and convolutions:

Suppose $j$ and $h$ are measurable functions on $\mathbb{R}$ whose Laplace transforms converge on some common vertical line $\operatorname{Re}(z)=\sigma$. Then the convolution $j * h$ is defined almost everywhere on $\mathbb{R}$, its Laplace transform converges on the same vertical line $\operatorname{Re}(z)=\sigma$ and satisfies $L_{j * h}(z)=L_{j}(z)$. $L_{h}(z)$ for $\operatorname{Re}(z)=\sigma$. Furthermore, if $\int_{-\infty}^{\infty}\left|L_{j}(\sigma+i t) \cdot L_{h}(\sigma+i t)\right| d t<\infty$, then the inversion formula

$$
(j * h)(t)=\frac{1}{2 \pi i} \int_{\sigma-i \infty}^{\sigma+i \infty} \mathrm{e}^{z t} L_{j}(z) \cdot L_{h}(z) d z
$$

holds for almost all $t \in \mathbb{R}$.

This follows from well-known properties of the Fourier transform (applied to $\mathrm{e}^{-\sigma t} j(t)$ and $\left.\mathrm{e}^{-\sigma t} h(t)\right)$.

Define

$$
\begin{aligned}
& J(t):=\frac{1}{2 \pi i} \int_{1-i \infty}^{1+i \infty} \mathrm{e}^{z t} \Gamma(z) d z=\exp \left(-\mathrm{e}^{-t}\right), \\
& H(t):=\frac{1}{2 \pi i} \int_{1-i \infty}^{1+i \infty} \mathrm{e}^{z t} \Gamma\left(z+\frac{1}{2}\right) d z=\mathrm{e}^{-t / 2} \exp \left(-\mathrm{e}^{-t}\right),
\end{aligned}
$$

where we have used (3.3) to explicitly evaluate the inverse Laplace transforms above. As we saw in (2.1), both $J$ and $H$ are $\mathrm{PF}_{2}$.

The Laplace transforms of both $J$ and $H$ converge for $\operatorname{Re}(z)>0$ and are given by

$$
L_{J}(z)=\Gamma(z), \quad L_{H}(z)=\Gamma\left(z+\frac{1}{2}\right) \quad(\operatorname{Re}(z)>0) .
$$

Hence the multiple convolution product

$$
f(t)=f_{r_{1}, r_{2}}(t):=\left(J^{*\left(r_{1}+r_{2}-1\right)} * H^{* r_{2}}\right)(t)
$$

converges for almost all $t \in \mathbb{R}$. It, of course, converges for all real $t$. A painless way to prove this is to remark that $H_{1}(t):=\mathrm{e}^{-t} H(t)$ and $J_{1}(t):=\mathrm{e}^{-t} J(t)$ are Schwarz functions on $\mathbb{R}$ and that $\left(J_{1}^{*\left(r_{1}+r_{2}-1\right)} *\right.$ $\left.H_{1}^{* r_{2}}\right)(t)=\mathrm{e}^{-t} f(t)$, as we saw in (2.4). We conclude from the composition property in Section 2 that $f$ is $\mathrm{PF}_{2}$. This fact will be essential in the proof of Theorem 2 .

The inversion formula (3.5) yields

$$
f(t)=\frac{1}{2 \pi i} \int_{1-i \infty}^{1+i \infty} \mathrm{e}^{z t} \Gamma(z)^{r_{1}+r_{2}-1} \Gamma\left(z+\frac{1}{2}\right)^{r_{2}} d z
$$


for almost all $t \in \mathbb{R}$. Here we need Stirling's formula to show that the above integral exists. In fact, Stirling's formula shows that the righthand side is analytic in $t$ inside the horizontal strip $|\operatorname{Im}(t)|<(n-1) \pi / 2$. Since the left-hand side is already known to be continuous $\left(\mathrm{e}^{-t} f(t)\right.$ being a Schwarz function), if follows that (3.7) holds for all $t \in \mathbb{R}$.

Now shift the contour of integration in (3.7) to the vertical line $\operatorname{Re}(z)=$ $-\frac{1}{4}$. This yields $|f(t)-p(t)| \leq C \mathrm{e}^{-t / 4}$, where $p(t)$ is the residue at $z=0$ of $\mathrm{e}^{z t} \Gamma(z)^{r_{1}+r_{2}-1} \Gamma\left(z+\frac{1}{2}\right)^{r_{2}}$, and

$$
C=\frac{1}{2 \pi} \int_{-\infty}^{\infty}\left|\Gamma\left(\frac{-1}{4}+i r\right)^{r_{1}+r_{2}-1} \Gamma\left(\frac{1}{4}+i r\right)^{r_{2}}\right| d r
$$

As $p(t)$ is a polynomial of degree $r_{1}+r_{2}-2$ in $t$ (vanishing if $r_{1}+r_{2}=1$ ), this shows $f(t) \leq C+|p(t)|$ for $t \geq 0$. As we have seen already that $\mathrm{e}^{-t} f(t)$ is bounded, we conclude that $f$ satisfies the hypotheses of Lemma 1 .

Let

$$
\begin{aligned}
q(t):=2 Q & \left(\pi^{-1} \mathrm{e}^{-t}\right) \\
& =\frac{1}{2 \pi i} \int_{1-i \infty}^{1+i \infty} \mathrm{e}^{z t}(4 z-1) \Gamma(z) d z=\left(4 \mathrm{e}^{-t}-1\right) \exp \left(-\mathrm{e}^{-t}\right) .
\end{aligned}
$$

Thus $q$ also satisfies the hypotheses of Lemma 1 . Hence $(f * q)(t)$ has at most one sign change for $t \in \mathbb{R}$. Comparing Laplace transforms, we find

$$
(f * q)(t)=2^{r_{1}}(2 \sqrt{\pi})^{r_{2}} g_{r_{1}, r_{2}}\left(\mathrm{e}^{-t} \pi^{-n}\right) \quad(t \in \mathbb{R}) .
$$

Hence $g(x)$ has at most one sign change for $x \in(0, \infty)$.

Next we show in a similar manner that each derivative $g^{(k)}(x)$ has at most one sign change for $x \in(0, \infty)$. Differentiating (3.2) $k$ times with respect to $x$ we obtain

$$
Q^{(k)}(x)=\frac{(-x)^{-k}}{4 \pi i} \int_{1-i \infty}^{1+i \infty}(\pi x)^{-z} \Gamma(z+k)(4 z-1) d z
$$

Similarly, differentiating (3.1) $k$ times with respect to $x$ we obtain

$$
g^{(k)}(x)=\frac{(-x)^{-k}}{c 2 \pi i} \int_{1-i \infty}^{1+i \infty}\left(\pi^{n} x\right)^{-z} \Gamma(z+k)(4 z-1) \Gamma(z)^{r_{1}+r_{2}-1} \Gamma\left(z+\frac{1}{2}\right)^{r_{2}} d z,
$$

where $c:=2^{r_{1}}(2 \sqrt{\pi})^{r_{2}}$. Thus $c g^{(k)}\left(\pi^{-n} \mathrm{e}^{-t}\right)$ is again the convolution of the $\mathrm{PF}_{2}$ function $f$ with $q_{k}(t):=2 Q^{(k)}\left(\pi^{-1} \mathrm{e}^{-t}\right)$, which has at most one change of sign for $t \in \mathbb{R}$. Lemma 1 again applies and shows that $g^{(k)}(x)$ has at most one sign change for $x \in(0, \infty)$. 
It still remains to prove that $g^{(k)}(x)$ has exactly one zero for $x \in(0, \infty)$ and that it is simple. So far we only know that the graph of $g^{(k)}$ crosses the $x$-axis at most once. We first show that $g(x)$ has at least one zero in $(0, \infty)$. By shifting the vertical integration contour in (3.1) to the left of the imaginary axis, we find [F1, p. 609, Remark iv] that $g(x)$ is negative in a non-empty interval $(0, \varepsilon)$. But the asymptotics of $g(x)$ as $x \mapsto+\infty$, worked out by Braaksma [Br, $\S 10]$, show that $g(x)>0$ for all large enough $x$. Alternatively, using just the arithmeticgeometric means inequality, Nils-Peter Skoruppa gave a simple proof that $g(x)>0$ for $x \geq(4 \pi)^{-n}[\mathbf{F 1}$, p. 609 , Remark vi]. Thus, $g$ has a zero in $\left(0,(4 \pi)^{-n}\right)$.

To prove our claim in Theorem 2 that $g$ has a unique and simple zero we shall use the following elementary fact.

Lemma 2. Suppose $h:(0, \infty) \mapsto \mathbb{R}$ is differentiable, vanishes at some $w_{0}$ but does not vanish identically on any open interval, satisfies $\lim _{x \rightarrow \infty} h(x)=0$, and suppose that the derivative $h^{\prime}$ changes sign at most once in $(0, \infty)$. Then $w_{0}$ is the only zero of $h$, and $h^{\prime}$ has a zero $w_{1}>w_{0}$.

Proof: Suppose $h(c)=0=h(d)$ for some $c<d$. After replacing $h$ by $-h$ if necessary, we may suppose that $h\left(x_{0}\right)>0$ for some $x_{0}$ strictly between $c$ and $d$. Comparing $h\left(x_{0}\right)$ with $h(c)$, and then with $h(d)$, we see by the mean-value theorem that there exist $y_{1}$ and $y_{2}$, with $c<y_{1}<$ $x_{0}<y_{2}<d$, satisfying $h^{\prime}\left(y_{1}\right)>0>h^{\prime}\left(y_{2}\right)$. Since $h^{\prime}$ changes sign at most once, we have $h^{\prime}(x) \leq 0$ for $x \geq d$, meaning that $h(x)$ is monotone decreasing for $x \geq d$. Since $\lim _{x \rightarrow \infty} h(x)=0=h(d)$, we must have $h(x)=0$ for all $x \geq d$. This contradicts our assumption that the zeroes of $h$ do not accumulate. Thus, $h$ could not have had two distinct zeroes $c$ and $d$, and so has a unique zero at $w_{0}$. But $h\left(w_{0}\right)=0$ and $h(x)$ tends to 0 as $x \rightarrow \infty$, so $h$ must assume a local maximum or minimum at some $w_{1}>w_{0}$. This $w_{1}$ is a zero of $h^{\prime}$.

Next we show that the hypotheses of Lemma 2 are fulfilled for $h=g^{(k)}$. We have already proved that $g^{(k+1)}$ has at most one sign change. The integral expression (3.10) shows that $\lim _{x \rightarrow \infty} g^{(k)}(x)=0$. Moreover, $g^{(k)}$ does not vanish identically since its Mellin transform does not. Using Stirling's formula, (3.10) implies that $g^{(k)}(x)$ extends to an analytic function of $x$ in a sector $\arg (x)<n \pi / 2$. Thus $g^{(k)}$ does not vanish identically on any open interval. Hence Lemma 2 and induction on $k$ imply that $g^{(k)}$ has a unique zero $z_{k}$, and that $z_{k+1}>z_{k}$. Since $z_{k} \neq z_{k+1}$, it follows that $z_{k}$ is a simple zero, concluding the proof of Theorem 2 . 
In Section 1 we showed how to deduce Theorem 1 from Theorem 2 and the behavior of $g(x)$ as $x \rightarrow 0^{+}$. There we assumed $r_{1}+r_{2}>1$ in order to ensure that this limit is $-\infty$. In the two signatures where $r_{1}+r_{2}=1$ the regulator is trivially 1 , but for the sake of completeness we remark that Theorem 1 still holds. The point is that we only need the limit (1.5) to be negative, and this still holds when $r_{1}+r_{2}=1[\mathbf{F} 1$, p. 609, Remark iv].

\section{References}

[Br] B. L. J. Branksma, Asymptotic expansions and analytic continuations for a class of Barnes-integrals, Compositio Math. 15 (1964), 239-341.

[Co1] H. CoHen, "A course in computational algebraic number theory", Graduate Texts in Mathematics 138, Springer-Verlag, Berlin, 1993.

[Co2] H. Cohen, "Advanced topics in computational number theory", Graduate Texts in Mathematics 193, Springer-Verlag, New York, 2000.

[F1] E. Friedman, Analytic formulas for the regulator of a number field, Invent. Math. 98(3) (1989), 599-622.

[Ka] S. KARLIN, "Total positivity", Vol. I, Stanford University Press, Stanford, Calif., 1968.

[Ma] J. Martinet, Petits discriminants des corps de nombres, in: "Number theory days, 1980" (Exeter, 1980), London Math. Soc. Lecture Note Ser. 56, Cambridge Univ. Press, Cambridge-New York, 1982, pp. 151-193.

[Od] A. M. ODLYZKo, Bounds for discriminants and related estimates for class numbers, regulators and zeros of zeta functions: a survey of recent results, Sém. Théor. Nombres Bordeaux (2) 2(1) (1990), 119-141.

[Po] M. Pohst, Eine Regulatorabschätzung, Special issue dedicated to the seventieth birthday of Erich Kähler, Abh. Math. Sem. Univ. Hamburg 47 (1978), 95-106.

[Re1] R. Remak, Über die Abchätzung des absoluten Betrages des Regulator eines algebraisches Zahlkörpers nach unten, J. reine angew. Math. 167 (1932), 360-378.

[Re2] R. REMAK, Über Grössenbeziehungen zwischen Diskriminante und Regulator eines algebraischen Zahlkörpers, Compositio Math. 10 (1952), 245-285. 
[Sch] I. J. Schoenberg, On Pólya frequency functions. II. Variation-diminishing integral operators of the convolution type, Acta Sci. Math. Szeged 12 (1950). Leopoldo Fejer et Frederico Riesz LXX annos natis dedicatus, Pars B, 97-106.

[Si1] J. H. Silverman, An inequality relating the regulator and the discriminant of a number field, J. Number Theory 19(3) (1984), 437-442.

[Zi] R. Zimmert, Ideale kleiner Norm in Idealklassen und eine Regulatorabschätzung, Invent. Math. 62(3) (1981), 367-380.

Departamento de Matemática

Universidad de Chile

Casilla 653

Santiago

Chile

E-mail address: friedman@uchile.cl 